\title{
Slip at the interface between immiscible polymer melts I: method to measure slip
}

\author{
Ryohei Komuro • Sathish K. Sukumaran • \\ Masataka Sugimoto $\cdot$ Kiyohito Koyama
}

Received: 22 April 2013 / Revised: 11 October 2013 / Accepted: 24 October 2013 / Published online: 14 November 2013

(C) The Author(s) 2013. This article is published with open access at Springerlink.com

\begin{abstract}
Slip at the interface between immiscible polymer melts remains poorly understood. A method that relies solely on rheological measurements to obtain the interfacial slip velocity uses the slip-induced deviation in the flow variables. To use the method, accurate estimates of the flow variables under the assumption of no-slip are necessary. Although such estimates can be easily derived under some cases, in general, this is not straightforward. Therefore, methods to determine the interfacial slip velocity without using estimates for the flow variables under no-slip conditions are desirable. In this work, we focus on investigations of slip at the interface between two immiscible polymer melts undergoing two-phase coaxial flow. To enable such investigations, we have adapted the Mooney method, usually used to investigate wall slip, to investigate polymer/polymer interfacial slip. Using this method, we have measured the slip velocity at the interface between polypropylene and polystyrene as a function of the interfacial stress. To determine the validity of the modified Mooney method, we also determine the slip velocity using the slip-induced deviation in the flow variables. To enable this determination, we use polypropylene and polystyrene with almost identical shear rate-dependent viscosities over a range of shear rates. The slip velocity obtained from the modified Mooney method displayed excellent agreement with that determined using the deviation from no-slip. In agreement with prior work, the dependence of the slip velocity on the interfacial stress is a power-law. Our investigation spans a sufficiently wide range of interfacial stress
\end{abstract}

R. Komuro · S. K. Sukumaran $(\bowtie) \cdot$ M. Sugimoto $\cdot$ K. Koyama Graduate School of Science and Engineering,

Yamagata University, 4-3-16 Jonan, Yonezawa,

Yamagata, 992-8510, Japan

e-mail: sa.k.sukumaran@gmail.com to enable the direct observation of two power-law regimes and also the transition between the two regimes. We also find that the power-law exponent of approximately 3 at low stresses decreases to approximately 2 at high stresses.

Keywords Interfacial slip - Immiscible polymer melt . Coextrusion - Capillary flow

\section{Introduction}

Typically, two chemically different polymers are immiscible, and the distinct phases are separated by interfaces. At the interface between two phases, the chemically different chains are likely to be weakly entangled. If entanglements are the main source of adhesion between the two components, then poor interfacial adhesion can be expected at the interface. During coextrusion of two immiscible polymeric liquids, as the flow rate is increased, the stress at the interface progressively increases. Due to the poor interfacial adhesion mentioned earlier, the interface would eventually be unable to support the interfacial stress. Under such conditions, one of the phases might slip with respect to the other. Such slip would also imply that the usually assumed no-slip boundary condition (Goldstein 1938) is no longer valid at such a liquid/liquid interface.

In spite of the rather simple scenario described above, the possibility of slip at the interface between two polymers remained rather controversial for a long time. At least part of the reason for the reluctance could be attributed to the success of the no-slip boundary condition in describing multiphase flows of Newtonian liquids (Goldstein 1938). However, during pressure-driven multilayer flow of polystyrene/polyethylene (both high density and low density), Han and Chin (1979) found that the pressure gradient 
at a given flow rate was even smaller than that of either of the two component polymers extruded individually. The authors hypothesized slip at the polymer/polymer interface in order to rationalize these puzzling results. Later, Bousmina et al. developed a model for the effective viscosity of immiscible blends of (generalized) Newtonian fluids that included the effect of slip at the liquid/liquid interface (Bousmina et al. 1999).

Among experimental techniques to study polymer/polymer interfacial slip, optical techniques have proved rather powerful (Migler et al. 2001; Lam et al. 2003; Zartman and Wang 2011). In such experiments, the slip velocity at the polymer/polymer interface can be obtained from the difference between the measured velocities of the incorporated particles that can be observed on either side of the polymer/polymer interface (Migler et al. 2001; Lam et al. 2003; Zartman and Wang 2011). Optical techniques are conceptually simple and yield accurate measurements of the slip velocity with few assumptions (Migler et al. 2001; Lam et al. 2003; Zartman and Wang 2011). However, the technique involves observing particles suspended in the flowing liquid and this requires equipment made of materials transparent to visible light. Given this, methods to determine the slip velocity at a liquid/liquid interface using only rheological measurements could prove useful.

Over the past decade, several groups have used rheological techniques to infer the slip velocity at a polymer/polymer interface (Zhao and Macosko 2002; Jiang et al. 2003; Lee et al. 2009; Park et al. 2010; Zhang et al. 2006). Most of these studies are restricted to multilayers with the parallel layers stacked on top of each other. For instance, Macosko and coworkers (Zhao and Macosko 2002; Lee et al. 2009; Park et al. 2010; Zhang et al. 2006) have estimated interfacial slip velocities using the reduction in the measured viscosity in the presence of slip when compared to the value estimated in the absence of slip. The viscosity in the absence of slip was determined as the volume fraction weighted harmonic mean of the viscosities of the individual polymers. While this estimate for the no-slip viscosity is appropriate for a stack of parallel multilayers, devising such estimates for other relevant geometries (such as coaxial flow during coextrusion) is not straightforward. The mixing rules (Utracki 1989; Bousmina et al. 1999) that are used for such estimations are usually at least partly empirical and their range of validity and accuracy are not always clear. Hence, methods for the determination of the slip velocity at a polymer/polymer interface directly from rheological data are few, especially for cases where reliable estimation of the noslip viscosity is not straightforward. This lack is particularly troublesome in the case of coextrusion because strain rates significantly higher than that during plane Couette steady shear flow (Lee et al. 2009; Park et al. 2010) are routinely accessed.
In this first of a series of planned articles, we propose a novel method to estimate the slip velocity at a polymer/polymer interface during two-phase coaxial flow. The method is a modification of the Mooney method and allows an approximate but sufficiently accurate determination of the slip velocity at the interface. To determine the validity of our method, we compare the slip velocity determined using the modified Mooney method to that obtained using the reduction in the viscosity due to slip when compared to the value obtained assuming no-slip. To render the estimation of the viscosity under the assumption of no-slip straightforward, we choose isotactic polypropylene (PP) and atactic polystyrene (PS) with almost identical viscoelastic properties over a range of shear rates (see Fig. 1). We then provide a comparative discussion of the results obtained from the two methods and finally, conclude.

\section{Materials and methods}

\section{Materials}

The materials chosen for the experimental study were PP $\left(M_{\mathrm{w}}=345,000 \mathrm{~g} / \mathrm{mol}, M_{w} / M_{n}=5.02\right)$ and PS $\left(M_{w}=\right.$ $\left.312,000 \mathrm{~g} / \mathrm{mol}, M_{w} / M_{n}=2.66\right)$. PP was kindly supplied by Japan Polypropylene Corporation and the PS by PS Japan Corporation. Commercial samples usually contain additives, and such additives can play a role in inducing slip i.e., they can act as lubricants. In order to avoid such confounding effects, we used specially prepared PP and PS samples that contain no additives except for a very small quantity of antioxidant.

\section{Rheological measurements}

Small amplitude oscillatory shear measurements were performed using a rotational rheometer (ARES, TA instruments) at $230{ }^{\circ} \mathrm{C}$ in a nitrogen atmosphere. Circular disks of each sample were prepared by compression molding at $230{ }^{\circ} \mathrm{C}$. The samples were sandwiched between parallel plates of $25 \mathrm{~mm}$ diameter and the gap was set at $1 \mathrm{~mm}$. Steady shear viscosity $\eta_{s}$ above a shear rate of $10^{2} \mathrm{~s}^{-1}$ was measured using a capillary rheometer (Capilograph, Toyo Seiki Seisakusho Ltd.). The wall shear stress values were corrected for capillary end effects by applying the Bagley correction to the pressure drop. The true wall shear rate was determined by applying the Weissenberg-Rabinowitsch correction to the apparent wall shear rate. Three different capillary dies, all with diameter, $D=1 \mathrm{~mm}$ and lengths, $L=$ 5, 10, and $20 \mathrm{~mm}$ were used. As can be seen from Fig. 1, when the corrections mentioned above were applied, we found that the data showed good agreement with the CoxMerz rule (Cox and Merz 1958). The solid lines in Fig. 1b 
a

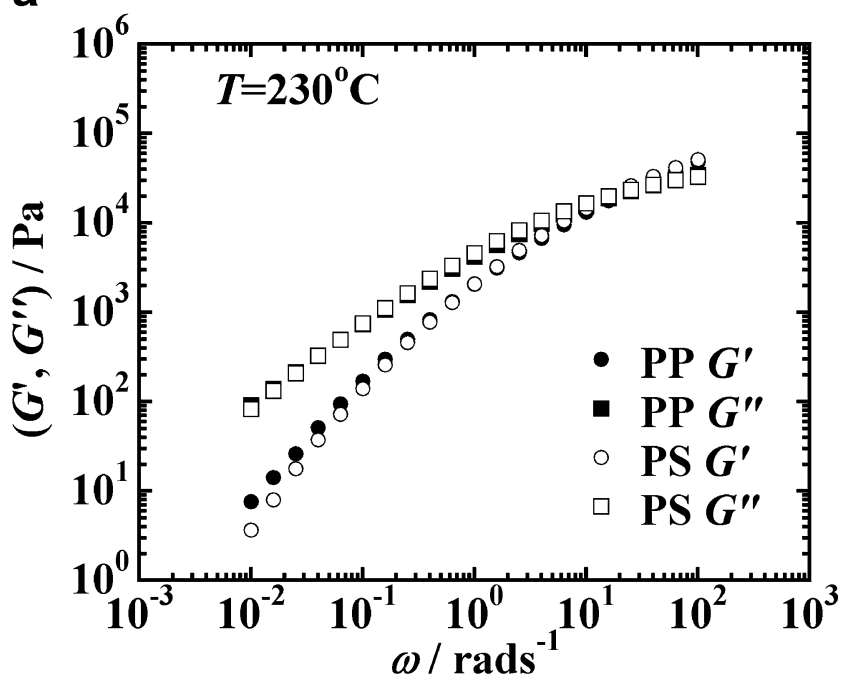

b

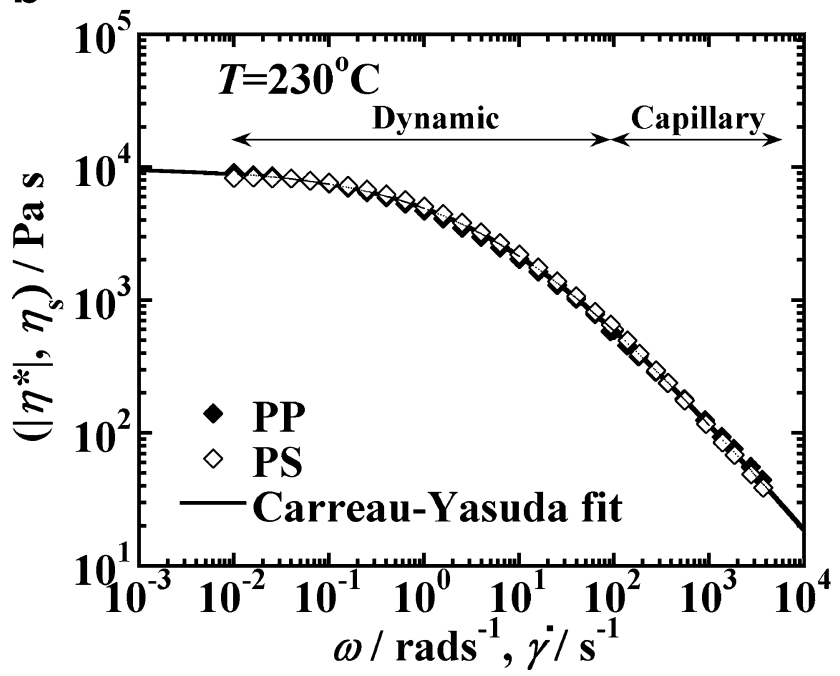

Fig. 1 a Storage modulus $G^{\prime}$ and the loss modulus $G^{\prime \prime}$ and $\mathbf{b}$ complex viscosity $\left|\eta^{*}\right|$ of PP and PS at $230^{\circ} \mathrm{C}$

are fits to the Carreau-Yasuda equation (Bird et al. 1977) for the viscosity dependence of the shear rate of PP and PS (see Eq. 1). The parameters of Carreau-Yasuda fit in Fig. $1 \mathrm{~b}$ are $\eta_{0}=8595$ Pa s, $n=0.33, \lambda=0.58$, and $a=0.69$.

The interfacial slip velocity was measured using capillary dies of three different diameters $D=1.0,1.5$, and $2.0 \mathrm{~mm}$. In order to minimize end pressure loss, the lengths of the capillaries were chosen such that for all the dies, the length to diameter ratio, $L / D=40$ (Kamal and Nyun 1980 ) and the entry angle was fixed at $60^{\circ}$ (Mitsoulis and Hatzikiriakos 2003). To minimize wall slip, capillary dies made of stainless steel were used (Larrazabal et al. 2006).

In order to realize the two-phase coaxial flow profile shown in Fig. 2, the concentric core-sheath samples were prepared by the following procedure. First, PP rods were produced by injection molding. Then, to coat the PS on the outside, each PP rod was dipped in a PS/tetrahydrofuran (THF) solution. Finally, after the evaporation of the THF under ambient conditions, all of the concentric core-sheath samples were vacuum-dried at $80^{\circ} \mathrm{C}$ for 2 days. The PS sheath was found to be of essentially uniform thickness of approximately $0.11 \mathrm{~mm}$ for all of the samples studied. The diameter of each concentric core-sheath rod was such that the sample was a close fit in the barrel of the capillary rheometer (barrel diameter $=9.55 \mathrm{~mm}$ ). The ratio of the PS thickness to the radius of the barrel of the capillary rheometer, $\left(R-R_{\mathrm{i}}\right) / R$, was approximately $2.3 \times 10^{-2}$. The length of the concentric core-sheath sample was $210 \mathrm{~mm}$.

It would of course be interesting to investigate the effect of switching the locations of the two polymers on interfacial slip. For this, we would need concentric core-sheath samples of the type used in this study but with PP as sheath and PS as the core. This, however, is not straightforward as it is rather difficult to coat a thin layer of PP on a core of PS. Therefore, in this study, we have not investigated interfacial slip for core-sheath samples with the polymers reversed, i.e., PP on the outside and PS on the inside. In general, the procedure used in this manuscript can be applied only to cases where a sufficiently thin layer of one polymer can be coated on another polymer such that concentric coresheath samples with the appropriate sheath thickness can be prepared.

Experimental estimation of the polymer/polymer interfacial slip velocity in two-phase coaxial flow

Figure 2 shows a schematic illustration of the cross section of the concentric core-sheath rod shaped sample (PP coated with PS) (subpanel a) and a schematic of the flow velocity distribution during core-sheath coextrusion in a cylindrical die (subpanel b). In order to enable easy determination of the slip velocity using the assumption of no-slip, we used the Carreau-Yasuda equation to fit the shear viscosity, $\eta$ versus shear rate, $\gamma$ data (Bird et al. 1977). The equation reads:

$\eta=\eta_{0}\left\{1+(\lambda \dot{\gamma})^{a}\right\}^{\frac{(n-1)}{a}}$,

where $\eta_{0}, \lambda, n$, and $a$ are the zero shear viscosity, time constant, power-law exponent, and a dimensionless parameter, respectively. For fully developed Poiseuille flow, the following equation holds:

$\frac{\sigma_{\mathrm{w}}}{R}=\frac{\sigma_{\mathrm{i}}}{R_{\mathrm{i}}}=\frac{\sigma(r)}{r}$,

where $\sigma_{\mathrm{w}}$ and $\sigma_{\mathrm{i}}$ are the shear stress values at the wall and the PP/PS interface, respectively. The radius of the core (PP) is $R_{\mathrm{i}}$. The shear rate in the capillary die is:

$\dot{\gamma}(r)=-\frac{\partial V(r)}{\partial r}(0 \leq r \leq R)$, 

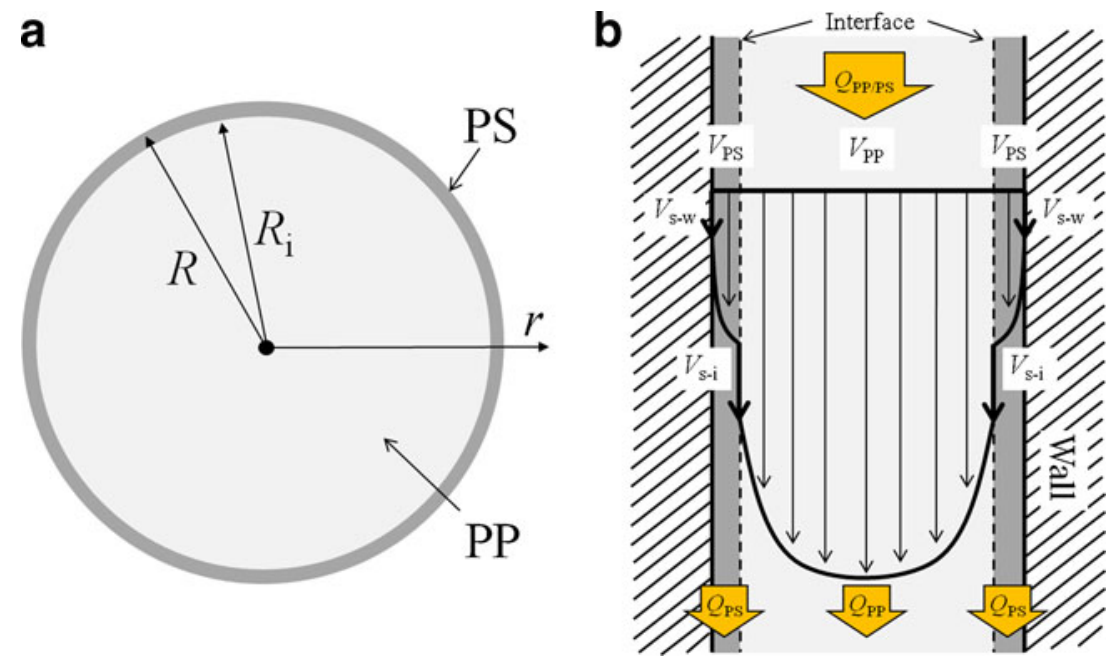

Fig. 2 a Cross section of PP coated with PS and $\mathbf{b}$ flow velocity profile during two-phase coaxial flow

where $V$ is the velocity field. Then, the flow velocity of the core $\left(V_{\mathrm{PP}}^{(\mathrm{C}-\mathrm{Y})}(r),\left(0 \leq r \leq R_{\mathrm{i}}\right)\right)$ and the sheath $\left(V_{\mathrm{PS}}^{(\mathrm{C}-\mathrm{Y})}(r),\left(R_{\mathrm{i}} \leq r \leq R\right)\right)$ can be evaluated using Eqs. 1-3. Whenever wall slip is negligible, the polymer/polymer interfacial slip velocity $V_{\mathrm{s}-\mathrm{i}}$ can be estimated from the deviations in the flow variables when compared to the values estimated assuming no-slip. For a single interface, the following equation is applicable:

$\left\{\begin{array}{l}V_{\mathrm{s}-\mathrm{i}}=\frac{Q_{\mathrm{PP} / \mathrm{PS}}-Q_{\text {no-slip }}}{\pi R_{\mathrm{i}}^{2}} \\ Q_{\text {no-slip }}=\int_{0}^{R_{\mathrm{i}}} 2 \pi r V_{\mathrm{PP}}^{(\mathrm{C}-\mathrm{Y})}(r) d r+\int_{R_{\mathrm{i}}}^{R} 2 \pi r V_{\mathrm{PS}}^{(\mathrm{C}-\mathrm{Y})}(r) d r\end{array}\right.$

where $Q_{\mathrm{PP} / \mathrm{PS}}$ and $Q_{\text {no-slip }}$ are the volumetric flow rate of the PP/PS samples and the volumetric flow rate of the PP/PS samples under no-slip, respectively. As mentioned earlier and shown in Fig. 1b, the PP and PS used in this study have similar shear rate-dependent viscosities at $230{ }^{\circ} \mathrm{C}$. Further, the Carreau-Yasuda equation could effectively describe the PP data (to within $2 \%$ ) and the PS data (to within $3 \%$ ). Therefore, the fitted Carreau-Yasuda function can be used to easily determine the shear rate-dependent viscosity under no-slip (see Fig. 1b).

The task of determining $Q_{\text {no-slip }}$ for use in Eq. 4 was rendered straightforward in our case by the judicious choice of PP and PS with matched shear-thinning characteristics. Determining $Q_{\text {no-slip }}$ for two-phase coaxial flow when the viscosities of the two polymers are different is not straightforward and as far as we are aware, no general procedure exists. Therefore, it would be highly desirable to devise a method to estimate $V_{\mathrm{s}-\mathrm{i}}$ for multiphase coaxial flows that does not require any no-slip data as input in Eq. 4. It turns out it is possible to devise such a method.
The desired method by which the interfacial slip velocity can be determined is a modification of the Mooney method (Mooney 1931). The modification relies on the fact that $R_{\mathrm{i}} \approx R$ for the core-sheath samples studied here. The Mooney method is traditionally used to determine the wall slip velocity (Mooney 1931). As detailed below, the modified Mooney method can be used to determine $V_{\mathrm{s}-\mathrm{i}}\left(=V_{\mathrm{PP}}\left(R_{\mathrm{i}}\right)-V_{\mathrm{PS}}\left(R_{\mathrm{i}}\right)\right)$ without the need for any information about the no-slip case.

Accounting for the possibility of slip at the wall and at the polymer/polymer interface, $Q_{\mathrm{PP} / \mathrm{PS}}$ can be written as

$$
\begin{aligned}
Q_{\mathrm{PP} / \mathrm{PS}}=\pi\left[R_{\mathrm{i}}^{2} V_{\mathrm{S}-\mathrm{i}}\right. & +R^{2} V_{\mathrm{s}-\mathrm{w}}+\int_{0}^{R_{\mathrm{i}}} r^{2}\left(-\frac{d V_{\mathrm{PP}}(r)}{d r}\right) d r \\
& \left.+\int_{R_{\mathrm{i}}}^{R} r^{2}\left(-\frac{d V_{\mathrm{PS}}(r)}{d r}\right) d r\right]
\end{aligned}
$$

where $V_{\mathrm{s}-\mathrm{w}}$ is the wall slip velocity. For fully developed Poiseuille flow $\left(\sigma_{\mathrm{w}} / R=\sigma_{\mathrm{i}} / R_{\mathrm{i}}=\sigma(r) / r\right)$, (5) can be rewritten,

$$
\begin{gathered}
Q_{\mathrm{PP} / \mathrm{PS}}=\pi\left[R_{\mathrm{i}}^{2} V_{\mathrm{s}-\mathrm{i}}+R^{2} V_{\mathrm{s}-\mathrm{w}}+\frac{R_{\mathrm{i}}^{3}}{\sigma_{\mathrm{i}}^{3}} \int_{0}^{\sigma_{\mathrm{i}}} \sigma^{2}\left(-\frac{d V_{\mathrm{PP}}}{d r}\right) d \sigma\right. \\
\left.+\frac{R^{3}}{\sigma_{\mathrm{w}}^{3}} \int_{\sigma_{\mathrm{i}}}^{\sigma_{\mathrm{w}}} \sigma^{2}\left(-\frac{d V_{\mathrm{PS}}}{d r}\right) d \sigma\right] .
\end{gathered}
$$

Equation 5 being generally applicable can be used to determine $V_{\mathrm{s}-\mathrm{i}}$ provided $R_{\mathrm{i}}$ and $\sigma_{\mathrm{i}}$ can be independently measured and controlled. However, this is usually not possible and even if possible, not always desirable. Therefore, we have chosen the thickness of the outer PS layer to be significantly smaller than the radius of the capillary such that the inner radius, $R_{\mathrm{i}}$, can be approximated by the radius of 
the capillary die, $R$ (i.e., $R_{\mathrm{i}} \approx R$ ). As mentioned earlier, the ratio of the PS thickness to the radius of the capillary die $\left(R-R_{\mathrm{i}}\right) / R \approx 2.3 \times 10^{-2}$. If $R_{\mathrm{i}} \approx R$, it follows that $\sigma_{\mathrm{w}} \approx \sigma_{\mathrm{i}}$. Using Eq. 2, it is clear that the relative error in the stress that is introduced by this approximation is also negligible as $\left(\sigma_{\mathrm{W}}-\sigma_{\mathrm{i}}\right) / \sigma \mathrm{W}=\left(R-R_{\mathrm{i}}\right) / R \approx 2.3 \times 10^{-2}$. Therefore, the last term on the right can be neglected. In addition, if wall slip is negligible $\left(V_{\mathrm{s}-\mathrm{w}}=0\right)$, (6) reduces to,

$\frac{Q_{\mathrm{PP} / \mathrm{PS}}}{\pi R_{\mathrm{i}}^{3}}=\frac{V_{\mathrm{s}-\mathrm{i}}}{R_{\mathrm{i}}}+\frac{1}{\sigma_{\mathrm{i}}^{3}} \int_{0}^{\sigma_{\mathrm{i}}} \sigma^{2}\left(-\frac{d V_{\mathrm{PP}}}{d r}\right) d \sigma$.

Equation 7 can be used to determine $V_{\mathrm{s}-\mathrm{i}}$ in a procedure akin to that usually used to determine the wall slip velocity. Therefore, by varying $R_{\mathrm{i}}$ (or equivalently, $R$ ) at a constant $\sigma_{\mathrm{i}}$ (i.e., constant pressure drop) and $L / R$ ratio, and measuring $Q_{\mathrm{PP} / \mathrm{PS}}, V_{\mathrm{s}-\mathrm{i}}$ can be determined.

In this study, we compared the results for the polymer/polymer interfacial slip velocity obtained using the two methods: (a) deviation from no-slip-Eq. 4 and (b) modified Mooney method-Eq. 7.

\section{Results and discussions}

First, we will describe the deviation from no-slip method to obtain the slip velocity (method (a)). Figure 3 shows the flow curves for the two pure melts (PP and PS) and the core-sheath PP/PS samples along with the Carreau-Yasuda fit for the pure melts. In this study, we measured $V_{\mathrm{s}-\mathrm{i}}$ using capillary dies of three different diameters $D=1.0$, 1.5 and $2.0 \mathrm{~mm}$ but with fixed $L / D=40$ and an entry angle of $60^{\circ}$. Therefore, the shear stress and shear strain rate shown in Fig. 3 are apparent values (i.e, the Bagley

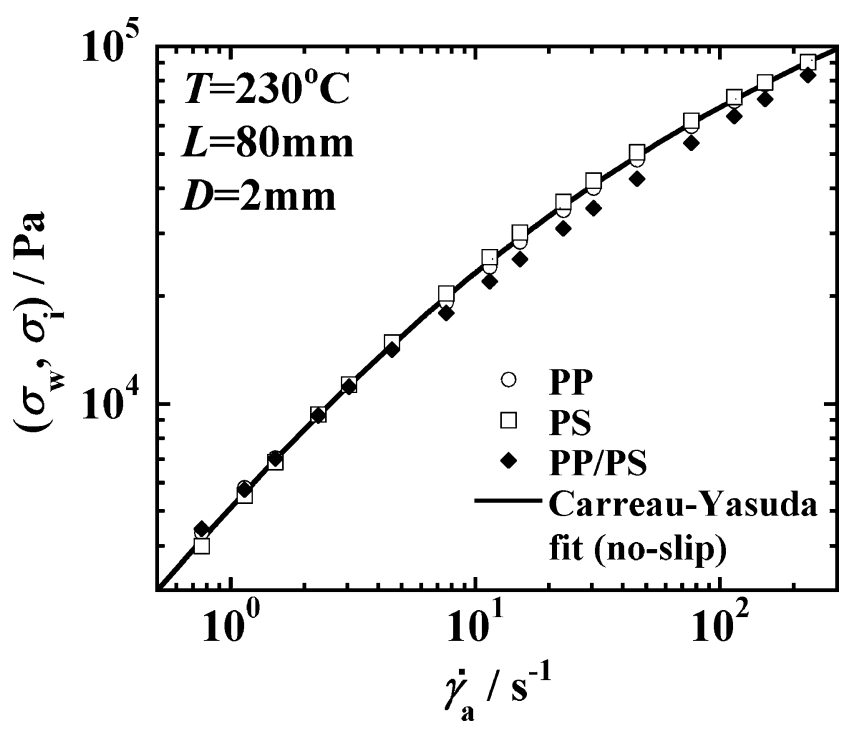

Fig. 3 Flow curve of PP, PS, PP/PS, the Carreau-Yasuda prediction for PP and PS correction and the Weissenberg-Rabinowitsch correction were not performed to determine the $\left.V_{\mathrm{s}-\mathrm{i}}\right)$. As the stress and strain rate are apparent values, the $\lambda$ and $a$ of the CarreauYasuda fit in Fig. 3 are slightly different from the parameters in Fig. 1b. The parameters for the Carreau-Yasuda fit shown in Fig. 3 are $\eta_{0}=8595 \mathrm{~Pa} \mathrm{~s}, n=0.33, \lambda=0.41$, and $a=0.71$. In order to evaluate the $V_{\mathrm{s}-\mathrm{i}}$ using the deviation from the no-slip condition (4), we used the parameters for the Carreau-Yasuda fit shown in Fig. 3. As Fig. 3 indicates, at low interfacial stress values, the apparent shear rates of the two-layer PP/PS samples display good agreement with that of the individual melts and the Carreau-Yasuda fit. However, above a certain critical shear stress, the apparent shear rate at a given value of shear stress deviates from the Carreau-Yasuda values indicating the existence of interfacial slip. Now, Eq. 4 can be used to determine the $V_{\mathrm{s}-\mathrm{i}}$ as a function of $\sigma_{\mathrm{i}}$ and the results are shown in Fig. 5.

To obtain $V_{\mathrm{s}-\mathrm{i}}$ using method (b), the quantity $Q_{\mathrm{PP} / \mathrm{PS}} / \pi R^{3}$ is plotted against $1 / R$ (which is equivalent to $1 / R_{\mathrm{i}}$ ) for various (constant) values of $\sigma_{\mathrm{w}}$ (or equivalently, $\sigma_{\mathrm{i}}$ or the pressure drop). Such plots are shown in Fig. 4a for PP, Fig. 4b for PS, and Fig. 4c for the core-sheath PP/PS samples. From Fig. 4a, b, it is clear that the slopes of the $Q_{\mathrm{PP}} / \pi R^{3}$ and $Q_{\mathrm{PS}} / \pi R^{3}$ versus $1 / R$ lines are both approximately zero. These results imply that for $\sigma_{\mathrm{W}}<10^{5} \mathrm{~Pa}$ there is negligible slip at the wall of the capillary die for both the PP and the PS melts. On the other hand, the slope of the line in Fig. $4 \mathrm{c}$ increases above a certain $\sigma_{\mathrm{i}}$. This suggests the existence of slip in the core-sheath PP/PS samples. As the slip at the wall is negligible for both the PP and the PS melts, the likely origin of the observed slip is at the interface between the PP and the PS in the core-sheath samples. This is also the reason for attributing the deviation in the shear rate from the Carreau-Yasuda values to slip at the polymer/polymer interface, as was done earlier. The data in Fig. $4 \mathrm{c}$ in combination with Eq. 7, which together constitute the modified Mooney method, can be used to determine $V_{\mathrm{S}-\mathrm{i}}$ from the slope of the $Q_{\mathrm{PP} / \mathrm{PS}} / \pi R_{\mathrm{i}}^{3}$ versus $1 / R_{\mathrm{i}}$ line. Using $\left(R-R_{\mathrm{i}}\right) / R \approx 2.3 \times 10^{-2}$ and propogating the error leads to a maximum relative error in the determination of the $V_{\mathrm{s}-\mathrm{i}}$ of approximately $4.5 \times 10^{-2}$. Consequently, the error bars in Fig. 5 are all smaller than the symbol size. This suggests that the error in the determination of the interfacial slip velocity introduced by the approximations inherent in the modified Mooney method are likely to be negligible and hence can be ignored.

While investigating wall slip, Hatzikiriakos et al. (1993) examined the effect of coating fluropolymers (FP) on the walls of a sliding plate rheometer on the observed slip of linear low density polyethylene (LLDPE) melts. The experimental procedure used by Hatzikiriakos et al. is conceptually similar to that used in our work but the flow geometry to which the method is applied is different. More 


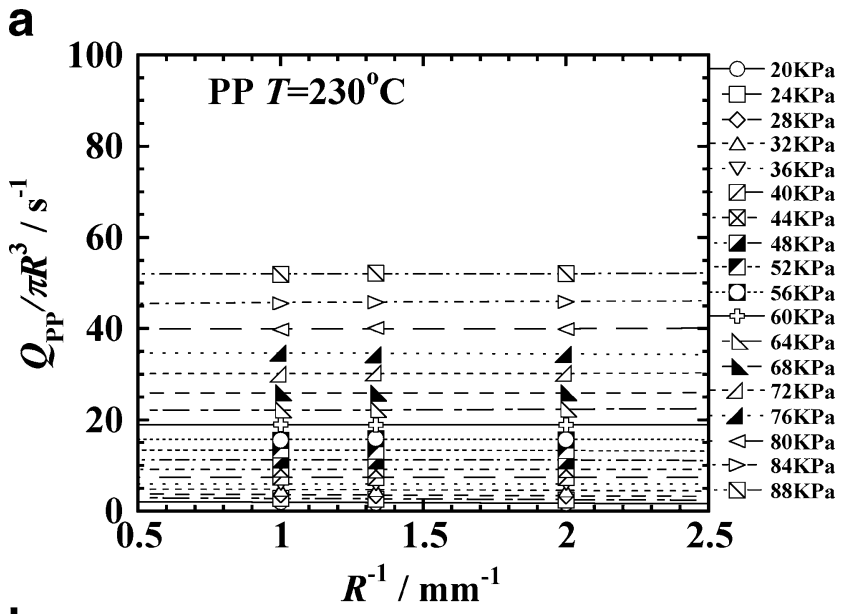

b

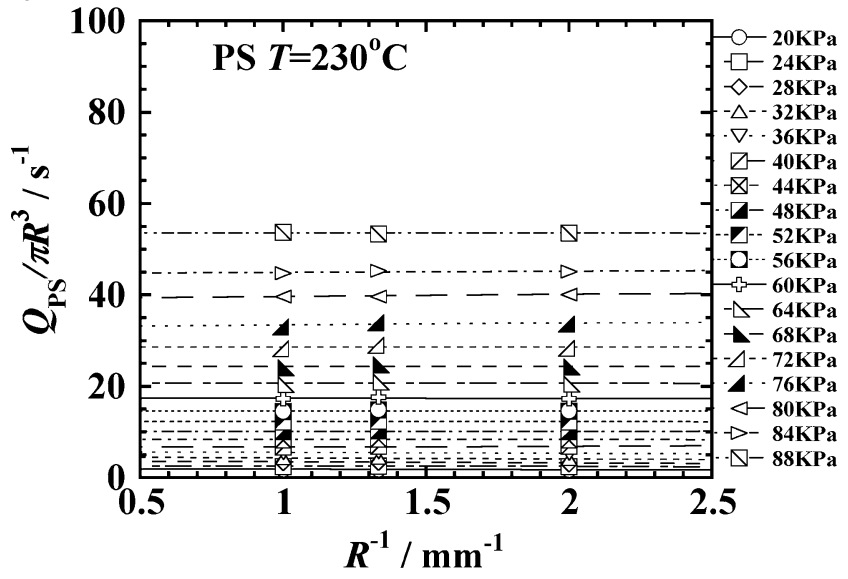

C

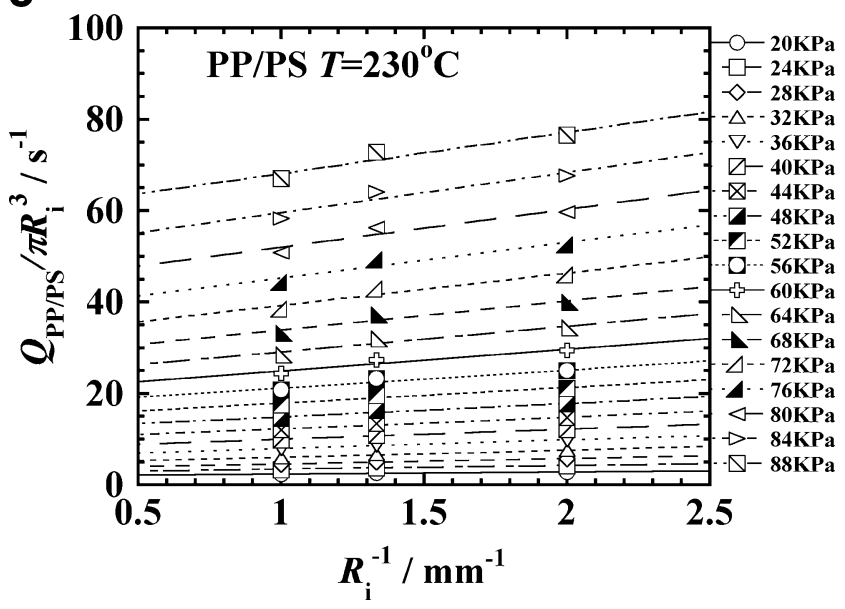

Fig. $4 Q / \pi R^{3}$ (and $Q_{\mathrm{PP} / \mathrm{PS}} / \pi R_{\mathrm{i}}^{3}$ ) as function of $1 / R$ (and $1 / R_{\mathrm{i}}$ ) for various constant $\sigma_{\mathrm{w}}$ (and $\sigma_{\mathrm{i}}$ ) for $\mathbf{a} \mathrm{PP}, \mathbf{b} \mathrm{PS}$, and $\mathbf{c} \mathrm{PP} / \mathrm{PS}$

importantly, in contrast to Hatzikiriakos et al., we exclusively focus on the slip that occurs at the interface between two immiscible polymers in the melt state. Further, in the experiments of Hatzikiriakos et al., the thickness of the FP layer, $h_{\mathrm{i}}$, was approximately $2 \mu \mathrm{m}$ and the gaps between the sliding plates, $H$, was either 0.36 or $0.23 \mathrm{~mm}$. This approximation contributes to a relative error in the gap

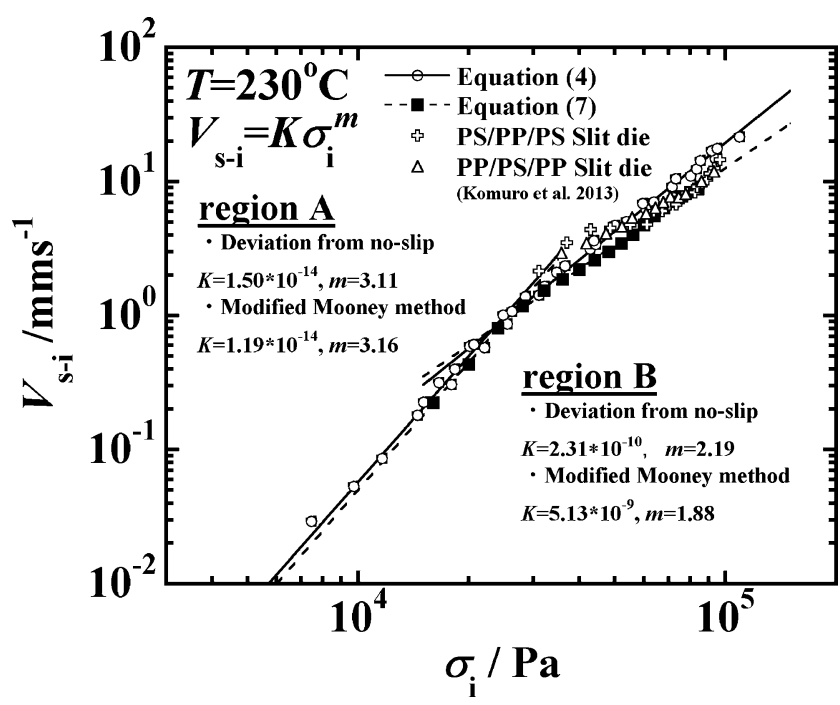

Fig. 5 Interfacial slip velocity $V_{\mathrm{s}-\mathrm{i}}$ as a function of interfacial shear stress $\sigma_{\mathrm{i}}$ for PP/PS from Eqs. 4 and 7, and the interfacial slip velocity $V_{\mathrm{s}-\mathrm{i}}$ for PS/PP/PS and PP/PS/PP obtained from Komuro et al. (2013). The error introduced by neglecting the PS layer is smaller than the symbol size

width, $\left(h_{\mathrm{i}}\right.$ (top) $+h_{\mathrm{i}}$ (bottom) $) / H \approx 1-2 \times 10^{-2}$. Interestingly, this relative error is comparable to the relative error in our work, $\left(\left(R-R_{\mathrm{i}}\right) / R \approx 2.3 \times 10^{-2}\right)$.

Figure 5 shows a comparison between the $V_{\mathrm{s}-\mathrm{i}}$ determined using Eq. 4 and that from Eq. 7. The figure suggests that, at a given $\sigma_{\mathrm{i}}, V_{\mathrm{s}-\mathrm{i}}$ determined from both Eqs. 4 and 7 are rather close. This suggests that Eq. 7 is an appropriate method to estimate the slip velocity at a polymer/polymer interface. The advantage of the modified Mooney method is that it can used to determine the interfacial slip velocity in core-sheath systems even when polymers with different shear rate-dependent viscosities are coextruded. Further, the slip at the PP/PS interface occurs at shear stress values that are significantly lower than the shear stress necessary for the onset of wall slip $\left(\approx 10^{5} \mathrm{~Pa}\right.$ ) (Hatzikiriakos and Dealy 1992; Mitsoulis et al. 2005; Komuro et al. 2010). If both wall slip and interfacial slip interfacial slip are present, it is not possible to determine the individual contributions to the total slip velocity using only the modified Mooney method. Hence, for the two polymers investigated here, the polymer/polymer interfacial slip velocity for $\sigma_{\mathrm{w}}>10^{5} \mathrm{~Pa}$ cannot be obtained.

It is also germane to note that the lower and upper limits of the range of $\sigma_{\mathrm{i}}$ over which the $V_{\mathrm{s}-\mathrm{i}}$ can be determined depend on both the diameter of capillary die and the pressure gradient. While using the deviation from no-slip method, i.e., Eq. 4, measurements from a single capillary die are sufficient to determine $V_{\mathrm{s}-\mathrm{i}}$. As we used three capillary dies with $D=1.0,1.5$, and $2.0 \mathrm{~mm}$, the range of $\sigma_{\mathrm{i}}$ over which the $V_{\mathrm{s}-\mathrm{i}}$ can be obtained is given by the union of the range of $\sigma_{\mathrm{i}}$ accessible by the individual dies and hence is 
relatively wide (see Fig. 5). On the other hand, while using the modified Mooney method, i.e., Eq. 7, $V_{\mathrm{s}-\mathrm{i}}$ for a given $\sigma_{\mathrm{i}}$ can be evaluated only when that $\sigma_{\mathrm{i}}$ is accessible by all three of the dies. Hence, the range of $\sigma_{\mathrm{i}}$ over which the $V_{\mathrm{s}-\mathrm{i}}$ can be measured using the Mooney method is given by the intersection of the range of $\sigma_{\mathrm{i}}$ that can be measured by each of the three dies and hence is relatively narrower (see Fig. 5).

Figure 5 indicates that the dependence of $V_{\mathrm{s}-\mathrm{i}}$ on $\sigma_{\mathrm{i}}$ is a power-law. Depending on the value of the interfacial stress, the power-law exhibits two distinct regimes with the power-law exponent changing from approximately 3 (region A) to approximately 2 (region B) at a critical interfacial stress of approximately $2 \times 10^{4} \mathrm{~Pa}$. The low-stress power-law exponent of 3 is in reasonable agreement with that found by Lee et al. (2009) for slip at the PP/PS interface. The high-stress exponent of 2 is similar to that found for a different polymer pair by Migler et al. using stroboscopic optical microscopy (Migler et al. 2001) and Park et al. using rheological measurements (Park et al. 2010). However, the actual $V_{\mathrm{s}-\mathrm{i}}$ values for PP/PS obtained in this study are consistently (approximately two to three times) larger than previous results (Zhao and Macosko 2002; Lee et al. 2009). A possible reason for the discrepancy is discussed below.

Recently, we also investigated polymer/polymer interfacial slip during coextrusion of a sandwich three-layer sample using a slit die (Komuro et al. 2013). These experiments were performed using the same set of samples used in the current study and at the same temperature $\left(230{ }^{\circ} \mathrm{C}\right)$. As discussed there, the power-law relationship between the interfacial slip velocity and the interfacial stress is essentially unchanged if the polymer in the inner layer is switched with that in the outer layer (Komuro et al. 2013). In addition, it was found that the interfacial slip velocity measured in the slit die using the PP/PS/PP and the PS/PP/PS layer stackings were rather close to the interfacial slip velocity measured in the capillary die using the concentric core-sheath PP/PS sample. These results suggest that the power-law relationship between $V_{\mathrm{s}-\mathrm{i}}$ and $\sigma_{\mathrm{i}}$ and the actual value of $V_{\mathrm{s}-\mathrm{i}}$ at a given $\sigma_{\mathrm{i}}$ are essentially independent of the flow geometry. In light of the independence of the value of the interfacial slip velocity on the flow geometry, one possible reason for the discrepancy in the $V_{\mathrm{S}-\mathrm{i}}$ values for PP/PS obtained in this study under two-phase coaxial flow and previous results in the literature on sandwich multilayer samples (Zhao and Macosko 2002; Lee et al. 2009) is the temperature at which the experiments were performed. As Zhao et al. and Lee et al. performed their experiments at $200{ }^{\circ} \mathrm{C}$, it is reasonable that our experiments at $230^{\circ} \mathrm{C}$ yield a higher interfacial slip velocity at a given interfacial stress. Preliminary measurements at $200{ }^{\circ} \mathrm{C}$ indicate that the interfacial slip velocity obtained from the modified Mooney method agree well with the values of Macosko and coworkers (Zhao and Macosko
2002; Lee et al. 2009). The effect of temperature on polymer/polymer interfacial slip will be further discussed in the planned third part of this series of articles.

Extrapolating the data of Lee et al. (2009) for PP/PS to where it intersects with the data of Migler et al. (2001) for polyethylene/fluoropolymer (PE/FP), the interfacial shear stress at the transition between the two power-law regimes can be estimated to be approximately $6 \times 10^{4} \mathrm{~Pa}$. Lee et al. (2009) also present data for PE/FP pair and using the high shear stress data of Migler et al. (2001) suggest that the transition occurs at $5 \times 10^{4} \mathrm{~Pa}$. In addition, independent measurements for a PE/FP interface by Park et al. (2010) found that the stress at the transition was approximately $5 \times 10^{4} \mathrm{~Pa}$. Although these experiments were performed using different polymer pairs, the value at the transition compares favorably with that determined from Fig. 5. When combined with our results, these results suggest that the value of the interfacial stress at the power-law transition is not sensitively dependent on the chemistry of the particular polymer pair being investigated. These results are certainly intriguing and we hope to shed additional light into this aspect in a future publication.

As pointed out in previous studies (Migler et al. 2001; Lee et al. 2009; Park et al. 2010), the values of the powerlaw exponent in the two regions disagree with existing theories including the classical predictions of (Brochard-Wyart and de Gennes 1993). In addition, the transition between the power-law regimes is a smooth crossover instead of the sudden jump envisaged in their predictions. The origin of the discrepancy is currently unclear. One reason suggested for the discrepancy between the theory and experiment is that all of the experiments, including ours, have used polymers with significant polydispersities. We hope that future studies using relatively monodisperse polymer pairs, if feasible, can shed light on this issue. Even without such direct corroboration of the theory, it is possible that the change in the power-law exponent between the two regimes corresponds to a change in the mechanism of slip at the polymer/polymer interface, similarly to that envisioned by Brochard-Wyart and de Gennes (1993). Investigations regarding the mechanisms of interfacial slip and also the effect of varying the viscosity of the two polymer components are also currently in progress.

\section{Summary}

We have investigated slip at a polymer/polymer interface during two-phase coaxial flow of PP and PS using two methods. To enable the determination of the slip velocity by measuring the deviation from no-slip, we choose two polymers with similar viscoelastic properties over a wide range of shear rates. On the one hand, such a choice drastically 
simplifies the estimation of viscosity of the concentric coresheath sample under no-slip. On the other hand, choosing two polymers with matched viscosities allows us to avoid considering possible effects of the viscosity difference on polymer/polymer interfacial slip. In addition, we have introduced a method to determine interfacial slip velocity in two-phase coaxial flow. The method is a modification of the Mooney method traditionally used to study wall slip. One advantage of this method is that, in principle, it is straightforward to apply even when the viscosities of the two polymers being coextruded are different. To establish the validity of the method, we compared the interfacial slip velocity determined by this method to that determined by the more conventional deviation from no-slip method. The slip velocity at the polymer/polymer interface $V_{\mathrm{s}-\mathrm{i}}$, as determined by the modified Mooney method agreed rather well with that determined by the deviation from no-slip. Further, slip at the polymer/polymer interface occurs at values of shear stress significantly lower than that necessary for the onset of wall slip $\left(\approx 10^{5} \mathrm{~Pa}\right)$.

The slip velocity at a polymer/polymer interface exhibits a power-law dependence on the interfacial shear stress. By measuring the interfacial slip velocity over a wide range of shear stress values, we were able to directly observe that the power-law exponent changes from approximately 3 to approximately 2 at a critical stress of approximately $2 \times$ $10^{4} \mathrm{~Pa}$. Combining the results of this study with that of Komuro et al. (2013), there exists strong evidence that neither the power-law relationship between $V_{\mathrm{s}-\mathrm{i}}$ and $\sigma_{\mathrm{i}}$ and nor the actual value of $V_{\mathrm{s}-\mathrm{i}}$ at a given $\sigma_{\mathrm{i}}$ strongly depend on the flow geometry.

Our results suggest that the modified Mooney method could be profitably used to estimate the slip velocity at a polymer/polymer interface. A significant advantage of the proposed method is that it can be used to investigate interfacial slip under two-phase coaxial flow where the two polymers possess different viscosities. Such an estimation is possible because the method does not rely on determining the volumetric flow rate under no-slip and consequently, mixing rules for the viscosity are not necessary.

Acknowledgments The authors gratefully acknowledge financial support from the Japan Society for Promotion of Science (JSPS) through Kakenhi (24350114) and the Dissemination of Tenure Tracking System Program of the Ministry of Education, Culture, Sports, Science and Technology - Japan. The authors also would like to acknowledge several fruitful discussions with T. Taniguchi of Kyoto University.

Open Access This article is distributed under the terms of the Creative Commons Attribution License which permits any use, distribution, and reproduction in any medium, provided the original author(s) and the source are credited.

\section{References}

Bird RB, Hassager O, Armstrong RC (1977) Dynamics of polymeric liquids, vol 1. Wiley

Bousmina M, Palierne JF, Utracki LA (1999) Modeling of structured polyblend flow in a laminar shear field. Polym Eng Sci 39:10491059

Brochard-Wyart F, de Gennes PG (1993) Sliding molecules at a polymer/polymer interface. C R Acad Sci Paris 317(II):13-17

Cox WP, Merz EH (1958) Correlation of dynamic and steady flow viscosities. J Polym Sci 28:619-622

Goldstein S (1938) Modern developments in fluid dynamics, vol 2. Oxford University Press

Han CD, Chin HB (1979) Theoretical prediction of the pressure gradients in coextrusion of non-Newtonian fluids. Polym Eng Sci 19:1156-1162

Hatzikiriakos SG, Dealy JM (1992) Wall slip of molten high density polyethylenes. II. Capillary rheometer studies. J Rheol 36:703741

Hatzikiriakos SG, Stewart CW, Dealy JM (1993) Effect of surface coatings on wall slip of LLDPE. Int Polym Proc 8:30-35

Jiang L, Lam YC, Yue CY, Yang YX, Tam KC, Li L, Hu XJ (2003) Energy model of the interfacial slip of polymer blends under steady shear. Appl Polym Sci 89:1464-1470

Kamal MR, Nyun H (1980) Capillary viscometry: a complete analysis including pressure and viscous heating effects. Polym Eng Sci 20:109-119

Komuro R, Kobayashi K, Taniguchi T, Sugimoto M, Koyama K (2010) Wall slip and melt-fracture of polystyrene melts in capillary flow. Polymer 51:2221-2228

Komuro R, Sukumaran SK, Sugimoto M, Koyama K (2013) Measuring slip at a polymer/polymer interface during three-layer flow. Nihon Reoroji Gakkaishi. J Soc Rheol Jpn 41:235-239

Lam YC, Jiang L, Yue CY, Tam KC, Li L, Hu X (2003) Interfacial slip between polymer melts studied by confocal microscopy and rheological measurements. J Rheol 47:795-807

Larrazabal HJ, Hrymak AN, Vlachopoulos J (2006) On the relationship between the work of adhesion and the critical shear stress for the onset of flow instabilities. Rheol Acta 45:705-715

Lee PC, Park HE, Morse DC, Macosko CW (2009) Polymer-polymer interfacial slip in multilayered films. J Rheol 53:893-915

Migler KB, Lavallee C, Dillon MP, Woods SS, Gettinger CL (2001) Visualizing the elimination of sharkskin through fluoropolymer additives: coating and polymer-polymer slippage. J Rheol 45:565-581

Mitsoulis E, Hatzikiriakos SG (2003) Bagley correction: the effect of contraction angle and its prediction. Rheol Acta 42:309-320

Mitsoulis E, Kazatchkov IB, Hatzikiriakos SG (2005) The effect of slip in the flow of a branched PP melt: experiments and simulations. Rheol Acta 44:418-426

Mooney M (1931) Explicit formulas for slip and fluidity. J Rheol 2:210-222

Park HE, Lee PC, Macosko CW (2010) Polymer-polymer interfacial slip by direct visualization and by stress reduction. J Rheol 54:1207-1218

Utracki LA (1989) Polymer alloys and blends. Hanser, Cincinnati

Zartman GD, Wang SQ (2011) A particle tracking velocimetric study of interfacial slip at polymer-polymer interfaces. Macromolecules 44:9814-9820

Zhang J, Lodge TP, Macosko CW (2006) Interfacial slip reduces polymer-polymer adhesion during coextrusion. J Rheol 50:41-57

Zhao R, Macosko CW (2002) Slip at polymer-polymer interfaces: rheological measurements on coextruded multilayers. J Rheol 46:145-167 